\title{
Integrin $\beta 3$ Targeting Biomaterial Preferentially Promotes Secretion of bFGF and Proliferation of iPSC-Derived Vascular Smooth Muscle Cells
}

\author{
Biraja C. Dash $^{1 *}$, Kaiti Duan ${ }^{1}$, Themis R. Kyriakides ${ }^{2,3,4}$, and Henry C. Hsia ${ }^{1,2 *}$
}

${ }^{1}$ Section of Plastic Surgery, Department of Surgery, Yale School of Medicine, Yale University, New Haven, CT 06510, USA

${ }^{2}$ Department of Biomedical Engineering, Yale University, New Haven, CT 06510, USA

${ }^{3}$ Department of Pathology, Yale University, New Haven, CT, 06510, USA

${ }^{4}$ Vascular Biology and Therapeutics Program, Yale University, New Haven, CT, 06510, USA

"Corresponding authors email: biraja.dash@yale.edu; henry.hsia@yale.edu;

Address: Yale University School of Medicine, 330 Cedar St., BB $3^{\text {rd }}$ Floor, PO Box 208041, New Haven, Connecticut 06510, Telephone: 203 737-2049, Fax: 203 785-5714 


\begin{abstract}
:
Human-induced pluripotent stem cell-derived-vascular smooth muscle cells (hiPSC-VSMC) have been shown to promote angiogenesis and wound healing. However, there is a paucity of research on how the extracellular matrix (ECM) microenvironment may impact the hiPSC-VSMC's function. In this study, our objective was to understand the effect of specific ECM ligand-integrin interaction on hiPSCVSMC's paracrine secretion, cell proliferation, and morphology. We here showed a precise modulation of hiPSC-VSMC in a fibronectin functionalized fibrillar collagen scaffold by targeting their integrin $\beta 3$. The secretion of proangiogenic growth factor, basic fibroblast growth factor (bFGF) was found to be fibronectin dependent via $\alpha v \beta 3$ integrin interactions. Also, our data indicate the possible role of a positive feedback loop between integrin $\beta 3$, bFGF, and matrix metalloproteinase- 2 in regulating hiPSCVSMC's morphology and cell proliferation. Finally, the secretome with improved proangiogenic activity shows potential for future regenerative applications.
\end{abstract}

Keywords: Induced pluripotent stem cell, vascular smooth muscle cell, integrin, paracrine factors, angiogenesis, fibronectin, bFGF 


\section{Introduction:}

Induced pluripotent stem cells (iPSC) with less immunogenic and ethical concerns are an incredible source for various cardiovascular cells $[1,2]$. These iPSC-derived cells mimic the phenotype and function of their in vivo counterparts and carry genetic information from patient. These promising attributes of iPSC technology have now been used to study disease and develop personalized medicine for various cardiovascular diseases $[3,4]$. One of the major cardiovascular cells that have been explored recently is vascular smooth muscle cells (VSMC) [5]. VSMC supports the formation of blood vessels and is involved in the pathophysiology of various vascular diseases [6]. Since the last decade, several advancements have been made to generate a large scale of VSMCs of the pure population with very high efficiency [7]. In one such effort, Patsch et al developed a protocol generating more than $80 \%$ pure population of VSMC in mere six days [8]. Furthermore, a groundbreaking method developed by Sinha and his colleagues showed differentiation of human iPSC (hiPSC) into developmental origin-specific SMC subtypes form neuroectoderm, lateral plate mesoderm, and paraxial mesoderm using a chemically defined protocol [9]. Similarly, we developed a protocol that can yield millions of hiPSC-VSMC in a matter of 2 weeks. Our protocol does not require a purification step while still leads to the production of more than $95 \%$ pure population of VSMC [10]. We further demonstrated that these cells are functionally similar to that of VSMC from lateral plate mesoderm (LM) embryonic origin [10].

The hiPSC-VSMC has shown promising results in the field of regenerative medicine and disease modeling $[1,5,7]$. Human iPSC-VSMC has been used in bioreactors and different scaffold systems to engineering larger blood vessels [11]. A recent report, by Luo et al, demonstrated the production of mechanically robust blood vessels [12]. We and others used hiPSC-VSMC derived from supravalvular aortic stenosis patients to study disease pathophysiology $[10,13,14]$. An elegant study by Misra et al. linked the lack of elastin production and hyperproliferation of SVAS-VSMC to overexpression of integrin $\beta 3$ [14]. Furthermore, another interesting report demonstrated that LM-derived hiPSC-VSMC is 
more proangiogenic compared to other subtypes of VSMC and set the foundation for our work on using these hiPSC-VSMC for wound healing [15]. We were the first to demonstrate the secretory function of this hiPSC-VSMC and the abundant number of paracrine factors released from these cells compared to adipose-derived stem cells (ADSC) $[16,17]$.

ECM based biomaterials have widely been used to modulate stem cell's secretome [18]. We recently reported that collagen fibrillar density is a potent modulator of paracrine secretion for hiPSCVSMC [16]. These ECM-based biomaterials initiate a ligand-integrin interaction and switch focal adhesion kinase on, which in turn activates a cascade of signaling that alter stem cell function [19]. Collagen $\square$ based scaffolds promote different regenerative processes via interactions with $\alpha 1 \beta 1$ and $\alpha 2 \beta 1$ integrin of cells. Besides collagen, fibronectin is a key ECM-based biomolecule in modulating stem cell function via $\alpha v \beta 3$ and $\alpha 5 \beta 1$ integrin [19]. Fibronectin with both integrin and growth factor-binding motifs in their protein sequence is an attractive biomolecule to engineer integrin-targeting scaffolds [20]. Recent studies have developed complex ECM system utilizing fibronectin and developing methods to present the fibronectin motifs accessible to binding to GF and fibronectins [20-22].

In this study, we sought to determine the effect of fibronectin and integrin $\beta 3$ interactions in a fibrillar collagen scaffold setting and whether this specific ligand-integrin signaling regulates paracrine secretion, morphology, and proliferation of hiPSC-VSMC (Figure 1). In particular, we examined the secretory profile of important proangiogenic, tissue remodeling, and anti-inflammatory factors produced by hiPSC-VSMC. Such factors may participate in angiogenesis and improve cell adhesion, proliferation, and migration of hiPSC-VSMCs. Furthermore, we investigated the mechanism of fibronectin mediated modulation of iPSC-VSMC. Our study suggests that fibronectin- $\alpha v \beta 3$ interaction activates a signaling cascade resulting in upregulation of bFGF, and MMP-2 and a positive feedback loop between integrin $\beta 3$, bFGF and MMP-2 to promote cell adhesion and proliferation of iPSC-VSMC. The current study 
suggests a strategy for precise modulation of the hiPSC-VSMC's secretome to improve cell-mediated regenerative healing.

\section{Methods:}

\section{Cell Culture:}

hiPSC-VSMC generated in a pure population using a previously established protocol were used for all the experiments [10]. The resulting differentiated cells were substantiated using immunofluorescence staining of SM-22 $\alpha$ and calponin, SMA, and SM-MHC to check purity. hiPSCVSMC of passage no more than 7 was used in the experiments. hiPSC-VSMC was grown and prepared on $1 \%$ gelatin-coated plates. The medium used smooth muscle growth media (SmGM)-2 with the addition of fetal bovine serum (FBS), with resultant FBS to be $10 \%$. The growth media was replenished every 48 hours, the iPSC-SMC was ready for usage after greater than $80 \%$ confluency. Human umbilical endothelial cell (HUVEC) was grown in EGM-2 medium and used after it reached more than $80 \%$ confluency.

\section{Fibronectin collagen scaffold production:}

The fibronectin functionalized collagen scaffold was constructed in the following steps. Briefly, collagen type-I of the initial concentration of $5 \mathrm{mg} / \mathrm{ml}$, fibronectin 1mg/ml, 10xMEM, and $1 \mathrm{M} \mathrm{NaOH}$ were mixed. The precise ratio of the listed ingredients for each of the formulations can be found in the supplementary table 1 . The varying degree of scaffold density was determined and manipulated by the amount of collagen present in the scaffolds. For lower density scaffolds, the rest of the volumes was made out using 1x PBS. For cell encapsulation in the scaffolds, cells were harvested using TrypLE at a concentration of $8000 / \mu 1$. 200,000 hiPSC-VSMC were then mixed in the neutralized slurry of fibronectin and collagen. The resultant mixture was then distributed as 100ul scaffold aliquots into 96 well cell culture plate. The plate was then incubated for 30 minutes at $37^{\circ} \mathrm{C}$ for the gelation. $200 \mu 1$ of 
Smooth muscle cell media was added on top of the solidified cell scaffolds and subsequently cultured for 3 days. At the end of the 72 hours, the conditioned medium was collected and used for all the downstream processing.

\begin{tabular}{|l|l|l|l|l|l|l|l|}
\hline Samples & Density & Collagen & $\mathbf{1 0 x}$ MEM & $\mathbf{1 M ~ N a O H}$ & $\begin{array}{l}\text { fibronectin } \\
\mathbf{1 m g} / \mathbf{m l}\end{array}$ & $\begin{array}{l}\text { SMC } \\
\text { media }\end{array}$ & $\begin{array}{l}\text { SMC } \\
\mathbf{8 k} / \mathbf{u l}\end{array}$ \\
\hline $\begin{array}{l}\text { CTRL- } \\
1.25 \mathrm{mg}\end{array}$ & $1.25 \mathrm{mg}$ & $125 \mathrm{ul}$ & $50 \mathrm{ul}$ & $2.4 \mathrm{ul}$ & $0 \mathrm{ul}$ & $300 \mathrm{ul}$ & $25 \mathrm{ul}$ \\
\hline $\begin{array}{l}\text { Fib } 25- \\
1.25 \mathrm{mg}\end{array}$ & $1.25 \mathrm{mg}$ & $125 \mathrm{ul}$ & $50 \mathrm{ul}$ & $2.4 \mathrm{ul}$ & $25 \mathrm{ul}$ & $275 \mathrm{ul}$ & $25 \mathrm{ul}$ \\
\hline $\begin{array}{l}\text { CTRL- } \\
2.5 \mathrm{mg}\end{array}$ & $2.5 \mathrm{mg}$ & $250 \mathrm{ul}$ & $50 \mathrm{ul}$ & $3.3 \mathrm{ul}$ & $0 \mathrm{ul}$ & $175 \mathrm{ul}$ & $25 \mathrm{ul}$ \\
\hline $\begin{array}{l}\text { Fib } 25- \\
2.5 \mathrm{mg}\end{array}$ & $2.5 \mathrm{mg}$ & $250 \mathrm{ul}$ & $50 \mathrm{ul}$ & $3.3 \mathrm{ul}$ & $25 \mathrm{ul}$ & $150 \mathrm{ul}$ & $25 \mathrm{ul}$ \\
\hline CTRL-4mg & $4 \mathrm{mg} / \mathrm{ml}$ & $400 \mathrm{ul}$ & $50 \mathrm{ul}$ & $8.4 \mathrm{ul}$ & $0 \mathrm{ul}$ & $25 \mathrm{ul}$ & $25 \mathrm{ul}$ \\
\hline Fib 25-4mg & $4 \mathrm{mg} / \mathrm{ml}$ & $400 \mathrm{ul}$ & $50 \mathrm{ul}$ & $8.9 \mathrm{ul}$ & $25 \mathrm{ul}$ & $0 \mathrm{ul}$ & $25 \mathrm{ul}$ \\
\hline Fib 50-4mg & $4 \mathrm{mg} / \mathrm{ml}$ & $400 \mathrm{ul}$ & $50 \mathrm{ul}$ & $8.9 \mathrm{ul}$ & $50 \mathrm{ul}$ & $0 \mathrm{ul}$ & $25 \mathrm{ul}$ \\
\hline
\end{tabular}

Table 1: Various formulations of collagen/fibronectin scaffolds.

\section{Integrin inhibition assay:}

The hiPSC-VSMC cultures in 2D and 3D scaffolds were incubated with echistatin and ATN-161, potent antagonists for $\alpha v \beta 3$ and $\alpha 5 \beta 1$ respectively [23, 24], at a concentration of $100 \mathrm{nM}$ concentration for $24 \mathrm{hr}$ on day 2 of culture. The cells and conditioned medium on day 3 were used to perform ELISA and AlamarBlue.

\section{Immunofluorescence staining:}

Human iPSC-VSMCs that were cultured on tissue culture plates and scaffolds were verified for phenotype characterization using anti-calponin, anti-SM-22 $\alpha$, anti-SMA, and anti-SMMHC primary antibodies. Selected samples were initially blocked with $5 \%$ BSA in $0.25 \%$ Triton X for 1 hour at room temperature. Authentication primary antibodies were added for incubation overnight at $4^{\circ} \mathrm{C}$. On the next day, the scaffolds were washed three times with PBST (tween20 0.05\%), and then the samples were incubated with secondary antibodies tagged with Alexa Fluor® 488 for 2 hours at room temperature. Dapi was used as a counterstain. $\mathrm{Z}$ stacks were taken at the interval $1 \mu \mathrm{m}$ and images were merged for the final image. Finally, the samples were washed in PBST three times at 5 minutes each before 
fluorescence imaging. The information related to primary and secondary antibodies can be found in tables $\mathrm{S} 1$ and $\mathrm{S} 2$.

\section{AlamarBlue assay:}

hiPSC-VSMC fibronectin induced collagen scaffolds were characterized for viability on day 3 of scaffold methodology as described. First, $200 \mu \mathrm{l}$ of culturing media was gently aspirated out for future ELISA assays. The scaffolds were then gently washed with $200 \mu 1$ of 1x PBS. AlamarBlue working solution was made at a 1:10 ratio of AlamarBlue stock to SmGM-2 medium. $100 \mu$ l of the AlamarBlue working solution was added to each of the scaffolds and incubated at $37^{\circ} \mathrm{C}$ for 2 hrs. After the incubation, the plate was read for Fluorescence intensity at $540 \mathrm{~nm}$ excitation and 590 emission wavelengths. Relative cell viability was evaluated by dividing them with the collagen scaffold fluorescence intensity value.

\section{Lactate dehydrogenase assay:}

Lactate dehydrogenase cytotoxicity assay was performed using an LDH cytotoxicity assay kit (Peirce, Thermofisher) as depicted $[16,25]$. Conditioned medium (CM) was used to determine the level of LDH in different samples. Groups that were included in this experiment were: CTRL, fib 25, and fib 50. Absorbance was measured at 490 and $680 \mathrm{~nm}$. To determine LDH activity, the 680nm absorbance value (background signal from the instrument) was subtracted from the $490 \mathrm{~nm}$ absorbance. Relative cytotoxicity value to control was determined.

\section{Live/Dead assay:}

Cell viability was also assessed via the Live/Dead assay as described. hiPSC-SMC scaffolds were incubated in PBS augmented with $4 \mu \mathrm{M}$ calcein-AM green (ThermoFisher, USA) and $2 \mu \mathrm{M}$ ethidium homodimer-1 (ThermoFisher, USA) for 30 minutes. Samples were visualized on an inverted Confocal Laser Scanning Microscope. $\mathrm{Z}$ stacks were performed at $1 \mu \mathrm{m}$ image intervals and images were merged for the final image. Four fields were obtained per scaffold and were imaged for each 
experiment. Viable and dead cells were counted using ImageJ software. The number of live and dead cells was then approximated.

\section{Qualitative ELISA:}

Day 3 culture media collected from the scaffolds were used to perform qualitative ELISA to assess for several pro-angiogenic growth factors VEGF, bFGF, MMP-2, Ang-1, TGF-beta, IL-10 SDF$1 \alpha$, and PDGF-AA released from hiPSC-VSMC as described $[16,25] .2 \mathrm{X} 90 \mu 1$ of the CM was placed to microwells of 96 well ELISA plates (NUNC MaxiSorp ${ }^{\mathrm{TM}}$ ) as duplicates. The plates were incubated at $4^{\circ} \mathrm{C}$ overnight. The next day, the plates were washed with PBST (PBS $+0.05 \%$ Tween-20) three times and blocked using 5\% BSA for 1 hour at room temperature. Then the plates were washed with PBST three times and were incubated with primary antibodies $(1: 2500)$ at $4^{\circ} \mathrm{C}$ overnight. The next day, the plates were washed three times with PBST and incubated with secondary antibodies conjugated with horseradish peroxidase (1:2500) for 2 hours at room temperature, avoid direct lighting whenever possible. The plates were then washed 3 times with PBST before adding $100 \mu$ of TMB substrate solution (Cell Signaling Technology, USA, Catalog: 7004S) to the plates after aspirating out the PBST and was incubated for 25 minutes at room temperature on a plate shaker. $100 \mu 1$ of stop solution (Cell Signaling Technology) was then added to each microwell and absorbance was measured at $450 \mathrm{~nm}$ on a plate reader. The information related to primary and secondary antibodies can be found in tables S1 and $\mathrm{S} 2$ in the supplementary information. The relative level of growth factor was evaluated by dividing them with the collagen scaffold absorbance value.

\section{Cell adherence and migration assay:}

Cell adherence assay for HUVECs was done in a 96 well plate. Briefly, 10, 000 cells were plated on a 96 well plate and cultured for 3 hours in CM supplemented smooth muscle basal medium (SmBM) of a ratio of 1:5. Controls were smooth muscle cell growth medium (SmGM-2), and SmBM. For the migration experiment, trans-well assays were performed using HUVECs as described (Dash et al., 2020). $1 \times 10^{4}$ cells were seeded on the top layer of the trans-wells ( $8 \mu \mathrm{m}$ pore size trans-wells, Falcon) 
and incubated for $10 \mathrm{~min}$ in cell culture incubators at $37^{\circ} \mathrm{C}$. The trans-wells were then placed into 24 well plates and $600 \mu 1$ of respective growth medium with $\mathrm{CM}$ were gently added to the wells from the inner side of the trans-well. The trans-wells were then kept in the cell culture incubator for 4 hours. Fixation was done with $100 \%$ cold methanol and gently removing the cells from the top layer. Transwells were washed and stained with Dapi. The number of cells migrated to the inner side of the membrane was then quantified by counting the number of Dapi stained nuclei from 4 different fields.

\section{In vitro network formation assay:}

The capability of hiPSC-VSMC-CM obtained from in situ scaffolds to induce angiogenesis was evaluated using an in vitro network formation assay as described [25, 26]. HUVECs were cultured until they were $80 \%$ confluent in an EGM-2 medium. $100 \mu 1$ of Matrigel (Corning Life Sciences) was used to evenly coat wells of 96 -wells plates. The plates were incubated for $30 \mathrm{~min}$ at $37^{\circ} \mathrm{C}$ to allow the Matrigel to solidify. HUVEC $\left(2 \times 10^{5} / \mathrm{ml}\right)$ that were dislodged in EGM-2 basal medium and $100 \mu$ l of this HUVEC containing solution along with $100 \mu 1$ of $C M$ were seeded in each well $(n=3)$. After 6 hours of incubation at $37^{\circ} \mathrm{C}$, any floating cells were then removed $\&$ the plates were washed twice with PBS and fixed using PFA for brightfield imaging. Images taken from 4 different fields/well were acquired and the number of nodes per field was calculated.

\section{Results:}

\section{Fibronectin mediates proliferation of hiPSC-VSMCs via $\alpha_{\mathrm{V}} \beta_{3}$ :}

Human iPSC-VSMC was generated using a previously established protocol [10]. The hiPSCVSMC was characterized for major smooth muscle cell markers calponin, SM-22 $\alpha$, SM-MHC, and SMA. The cells were highly positive for the aforementioned markers (Figure 2A). The cells were positive for SM-MHC but did not express mature fibers (2A). The hiPSC-VSMC was then seeded on fibronectin-coated plates to determine their proliferation. The cells on days 1, 2, and 3 proliferated on fibronectin and were significantly more compared to the control with no fibronectin (Figure 2B). Most importantly, the hiPSC-VSMC in control wells did not significantly proliferate over three days. 
Furthermore, the AlamarBlue assay showed a significantly reduced proliferation of hiPSC-VSMCs on fibronectin upon treatment with echistatin, a known antagonist of $\alpha_{\mathrm{V}} \beta_{3}$ (Figure 2C).

The hiPSC-VSMC was then embedded in a 3D collagen scaffold functionalized with fibronectin and was analyzed for cell proliferation, cytotoxicity, and phenotype after three days in culture (Figure 3). The collagen scaffold of $4 \mathrm{mg} / \mathrm{ml}$ final concentration was used with fibronectin of $50 \mu \mathrm{g} / \mathrm{ml}$ of final concentration. The AlamarBlue assay data showed an enhanced proliferation of hiPSC-VSMC in the fibronectin functionalized scaffolds (Figure 3A). The LDH assay was used to assess the cytotoxicity of the collagen/fibronectin scaffold and control collagen scaffolds. The LDH assay showed a minimal cytotoxic effect of collagen/fibronectin as compared to the control collagen scaffolds (Figure 3B). Furthermore, immunofluorescence staining was performed to characterize the morphology and phenotype of hiPSC-VSMC in the scaffolds (Figure 3C). Immunofluorescence images showed an elongated morphology of hiPSC-VSMC inside collagen/fibronectin scaffolds as compared to collagen control scaffolds. The hiPSC-VSMC maintained their smooth muscle cell phenotype in the hydrogels as shown by staining with calponin and SM-22 $\alpha$, SM-MHC, and SMA (Figure 3C) during three days of in vitro culture. Figure 3D shows immunofluorescence images of Calcein-AM ${ }^{+}$cells (live) and EthD- $1^{+}$ (dead) cells. The graphs showed an increased number of live hiPSC-VSMC in collagen/fibronectin scaffolds compared to control scaffolds (Figure 3D and E), whereas the ratios of the dead to the total number of cells were found to be similar in both the scaffolds (Figure 3F).

Furthermore, AlamarBlue assay showed a significantly reduced proliferation of hiPSC-VSMC in collagen/fibronectin scaffold in response to echistatin (Figure 3G), whereas we did not observe any effect of ATN-161, a potent inhibitor of $\alpha_{5} \beta_{1}$, on the proliferation of iPSC-VSMC in the collagen/fibronectin scaffold (Figure 3H).

\section{Fibronectin selectively upregulates bFGF secretion via $\alpha_{v} \beta_{3}$}


Qualitative ELISA-based analysis demonstrated the fold change of proangiogenic growth factors VEGF, bFGF, ANG-1, SDF-1 $\alpha$ and PDGF-AA, TGF $\beta$, MMP-2, and IL-10 in the CM of scaffolds (Figure 4A). The level of bFGF and MMP-2 was seen to be upregulated in the collagen/fibronectin scaffold. Especially, bFGF was seen to be enhanced 4-5 folds in the case collagen/fibronectin scaffolds compared to the control collagen scaffolds (Figure 4A). The relative level of bFGF was then investigated in collagen/fibronectin scaffolds across different densities of type I collagen. The data showed an enhanced level of bFGF in the higher density $(4 \mathrm{mg} / \mathrm{ml})$ of type I collagen (Figure 4B). Figure $4 \mathrm{C}$ shows an increase in the relative bFGF level with an increase in the amount of fibronectin from 10 to $100 \mu \mathrm{g} / \mathrm{ml}$ with $100 \mu \mathrm{g} / \mathrm{ml}$ releasing the most significant amount of bFGF, while $50 \mathrm{ug}$ had a sub-optimal increase in the bFGF. 10 and $25 \mu \mathrm{g} / \mathrm{ml}$ showed no difference and were similar to that of control collagen scaffolds. The treatment with echistatin showed a decrease in the bFGF secretion level compared to no treatment (Figure 4D). However, we did not see any effect of ATN-161 on bFGF release (Figure 4E).

We saw a comparatively enhanced relative level of MMP-2 secretion in $2.5 \mathrm{mg} / \mathrm{ml}$ and $4 \mathrm{mg} / \mathrm{ml}$ fibronectin functionalized collagen scaffolds (Figure 5A) compared to control collagen scaffolds. However, we did not see any difference in the MMP-2 secretion between 50 to $100 \mu \mathrm{g} / \mathrm{ml}$ of fibronectin (Figure 5B). Also, incubation with Echistatin and ATN-161 did not show any difference in the MMP-2 secretion in the cells embedded in collagen/fibronectin scaffolds. (Figure 5C and 5D ).

Furthermore, when we cross-linked the collagen/fibronectin scaffold with a PEG-based amine cross-linker, 4S-StarPEG, the release of bFGF was reduced to the level of control scaffolds (Figure 6A). A similar trend was seen for MMP-2 release as well (Figure 6B). There was no difference in the cell viability between cross-linked and non-crosslinked scaffold groups (Figure 6C). The cross-linking of amine groups was investigated and can be seen in figure S1 in the supplementary information. The 
TNBSA data quantifies free primary amine groups and can be seen lowering in the cross-linked group compared to the control (non-crosslinked).

\section{The conditioned medium from the fibronectin functionalized collagen scaffold promotes endothelial cell adhesion, migration, and network formation:}

Functional assays of the CM were carried out to determine the bioactivity of the secreted paracrine growth factors from the final collagen/fibronectin scaffolds (Figure 7) and were compared with SmGM-2, positive control endothelial cell growth medium-2 (EGM-2), and negative control endothelial basal medium (EBM). Cell adherence of HUVEC was found to be significantly more in CM compared to control SmGM-2, EGM-2, and EBM medium controls (Figure 7A and B). The HUVECs showed typical cobblestone-like morphology in the case of both CM and EGM-2 after $3 \mathrm{~h}$ of seeding (Figure 7A). A similar pattern can be seen in the case of migration of HUVECs towards CM in a transwell migration assay (Figure 7C and D). HUVECs in the case of SmGM-2 and EBM showed reduced migration compared to CM and EGM-2 (Figure 7C and D). And SmGM-2 had enhanced migration compared to the EBM control medium. In vitro network formation assay using HUVECs on Matrigel showed higher nodes/field in the case of both EGM-2 and CM and were significantly more than that of the SmGM-2 and EBM (Figure 7E and F). There was no difference between SmGM-2 and EBM, although an increase can be seen in the case of SmGM-2 it was not significant.

\section{Discussion:}

Human iPSC-derived VSMC has lately received major scientific attention in regenerative vascular research [7]. Recent attempts to generate robust implantable blood vessels and regenerate wounds have rendered these cells highly relevant for the development of functional vascular grafts and proangiogenic therapeutic modules $[12,16,17,27,28]$. An earlier study has implicated LM-VSMC in promoting angiogenesis [15]. We have taken a step forward and investigated the proangiogenic and 
immunomodulatory secretory function of this hiPSC-VSMC from LM embryonic origin for wound healing application $[16,17]$.

In our recent studies, we reported that the hiPSC-VSMC delivered in a collagen-based biomimetic scaffold promotes wound closure with enhanced vascular regeneration and reduced inflammation [16]. We attempted to understand how extracellular matrix such as collagen modulates their function. Our study led to the understanding of the effect of collagen fibrillar density on the paracrine secretion of hiPSC-VSMC. We showed that increase in collagen fibrillar density enhances the secretion of major proangiogenic factors such as vascular cell endothelial growth factor (VEGF), bFGF, angiopoietin (ANG)-1, platelet-derived growth factor (PDGF), transforming growth factor (TGF)- $\beta 1$, stromal cell-derived factor (SDF)-1 $\alpha$, and MMP-2 [16]. In another study, we tried to understand the effect of hyaluronic acid (HA) on the hiPSC-VSMC secretory function. The HA while promoted proliferation of hiPSC-VSMCs in a collagen scaffold did not show any effect on their secretory function $[25]$.

This sets the stage for developing strategies for precise modulation of hiPSC-VSMC's function. One of the approaches is the development of integrin-targeting biomaterials to modulate the cell's function for improved regenerative potential [19]. The method involves functionalization of the biomaterials with ECM biomolecules with a central idea that the ligand of the ECM biomolecules targets specific integrin interactions and regulate cellular responses, such as increased cell migration, proliferation, and paracrine secretion [22, 29, 30]. This hiPSC-VSMC from LM origin has earlier shown to be expressing integrin $\beta 3$ and was found to be highly upregulated in proliferative phenotype [14]. In this study, we functionalized the collagen scaffold with a biomolecule that can facilitate specific interaction with the integrin $\beta 3$ receptor. One of the natural choices was fibronectin. Fibronectin has two binding sites one is an integrin-binding site and the other is RGD or growth factor (GF) binding site next 
to it [20-22]. The simultaneous binding of GF and integrins can lead to a synergistic integrin/GF receptor signaling enhancing the effect of GF on hiPSC-VSMC.

Our initial screening on 2D tissue culture plates showed improvement in cell proliferation of hiPSC-VSMC in response to fibronectin. This was further validated using an antagonist of $\alpha v \beta 3$, echistatin. In a 3D setup fibronectin in a fibrillar collagen scaffold enhances cell proliferation and secretion of bFGF. We were able to reverse the rate of proliferation and bFGF secretion by inhibiting $\alpha v \beta 3$ integrin signaling. Fibronectin regulates the function of the cell through $\alpha 5 \beta 1$ as well. However, we did not see fibronectin regulating cell proliferation and bFGF secretion via $\alpha 5 \beta 1$ as shown by incubating with ATN-161, an antagonist of $\alpha 5 \beta 1$. Furthermore, the fibronectin regulates the cell proliferation and bFGF secretion in a collagen fibril density-dependent manner as shown by the $4 \mathrm{mg} / \mathrm{ml}$ collagen density with enhanced cell proliferation and bFGF secretion. Most importantly, the amount of fibronectin determined the proliferation and bFGF secretion. The higher the amount of fibronectin the better the response while the lower amount of fibronectin such as 10 and $25 \mu \mathrm{g} / \mathrm{ml}$ did not elicit the desired response. The cells were shown to be proliferative as shown in their elongated morphology in the fibronectin functionalized scaffold.

FGFs and their role in angiogenesis are very well studied. bFGF is one of the FGFs which acts as a potent inducer of angiogenesis [31]. bFGF positioned at the upstream in the signaling system interacts with growth factors and chemokines such as VEGF, PDGF, hepatocyte growth factor, and monocyte chemoattractant protein to contribute towards the development of mature vessels and collateral arteries [32]. We believe an elevated level of bFGF in the case of the CM of the fibronectin functionalized scaffold might be responsible for promoting cell attachment, migration, and network formation of HUVECs in vitro. bFGF is also known to interact with $\alpha v \beta 3$ integrin to promote vascular cell adhesion 
during angiogenesis [33]. We believe a similar mechanism might be existing here. bFGF and $\alpha v \beta 3$ might be creating a positive feedback loop for enhanced iPSC-VSMC cell adhesion.

MMPs, extracellular proteinase, are targets that regulate cell behavior and have recently been used to modulate stem cell function [34]. MMPs not only degrade structural proteins of ECM but also cleave cell surface molecules such as integrins to change cell function $[35,36]$. Numerous studies have shown cross-talk between MMP-2 and $\alpha v \beta 3$ to promote stem cell adhesion, proliferation, and migration [37]. In blood vessels, they are found to be colocalized [38]. MMP-2 has also been shown to cleave fibronectin into small fragments to enhance the adhesion and migration of human melanoma cells by $\alpha v \beta 3$ [39]. In this study, we found that in collagen scaffold higher than $1.25 \mathrm{mg} / \mathrm{ml}$ the MMP-2 is upregulated in the presence of fibronectin. We believe that the increase in MMP-2 might be facilitating an augmented interaction between fibronectin and $\alpha v \beta 3$ integrins present in iPSC-VSMCs [40]. Besides, MMP-2 is crucial in releasing bFGF from ECM to regulate cell migration [37, 41]. MMP-2 is also known to stimulate the interaction of VSMCs with newly formed ECM [42]. During pathophysiology's such as hypertension, this interaction between VSMC and ECM triggers in phenotypic switching from a contractile phenotype to a synthetic one for the cells to migrate [42]. So in other words release of MMP2 might be regulating the migration of iPSC-VSMC via integrin signaling and disrupting growth factors bound to ECM.

However, the exact mechanism of MMP-2 regulation in the fibronectin functionalized 3D collagen scaffold is not known. In the past, it has been shown that MMP-2 gene expression could be induced via activator protein-1 after integrin-linked kinase activation after cell integrin and fibronectin engagement [43]. However, our study with the integrin antagonists did not show any changes in the amount MMP-2 indicating any changes in the MMP-2 level is independent of integrin and fibronectin interaction. An earlier study shows a direct regulation of MMP-2 by bFGF in endothelial cells [44]. In 
another study on prostate cancer cells associated with prostate fibroblasts or osteoblasts showed enhanced MMPs in the presence of bFGF and TGF $\beta 1$ [45]. Nevertheless, the integrin inhibition assay which resulted in lower level bFGF was not enough to show any direct link between bFGF and MMP2. To completely block the expression of bFGF to determine the role of bFGF in MMP-2 secretion we adopted an engineering approach and cross-linked the fibronectin and collagen using a 4S-StarPEG nontoxic cross-linker that binds to free primary amine groups. Our idea was to make the binding motifs of fibronectin less accessible to integrin present in iPSC-VSMCs. The cross-linked resulted in a huge drop in the release of bFGF. We also saw a subsequent decrease in MMP-2 expression. This demonstrates that MMP-2 expression in hiPSC-VSMC is regulated by bFGF.

Finally, it is the combination of physical cues, the production of growth factors, and the MMPs that support hiPSC-VSMC proliferation in a fibronectin functionalized scaffold. Our study indicates the presence of a positive feedback loop between integrin $\beta 3$, bFGF, and MMP-2. We, therefore, propose an IGM cycle (Integrin- Growth factor-MMP) to account for the modulation of hiPSC-VSMC (Figure 8).

\section{Conclusion:}

In summary, we showed an approach to regulate the secretory function of hiPSC-VSMC by targeting their integrin $\beta 3$ receptor. The fibronectin within a collagen scaffold promoted bFGF secretion via $\alpha v \beta 3$ secretion and was dependent on the collagen fibrillar density and amount of fibronectin. Finally, we believe i) bFGF regulates the upregulation of MMP-2 in hiPSC-VSMCs in an integrin independent pathway and ii) a positive feedback loop between integrin $\beta 3$, bFGF, and MMP-2 exists and promotes iPSC-VSMC proliferation. Furthermore, bFGF in the CM showed proangiogenic potential by enhancing cell adherence, migration, and network formation of HUVECs. Ongoing efforts are to engineer complex ECM-based biomaterial systems for developing iPSC-based vasculature. 
Supplementary Information: The Supporting Information is available online and contains additional information on materials, antibodies, and TNBSA method and data.

Author Contributions: B.C.D. conceived the study. B.C.D. and H.C.H. procured the funding. B.C.D. designed the experiments. B.C.D. and K.D. performed the experiments. B.C.D. wrote the manuscript. All the authors participated in data analysis, discussed the results, and reviewed the manuscript.

Funding: This work was funded by the Plastic Surgery Foundation Grant 18-003032 (H.C.H and B.C.D) and Yale Department of Surgery (H.C.H).

Acknowledgment: The authors would like to thank the core research facilities at the Yale Department of Surgery.

Conflicts of Interest: The authors declare no conflict of interest. 


\section{References:}

[1] D. Klein, iPSCs-based generation of vascular cells: reprogramming approaches and applications, Cell Mol Life Sci 75(8) (2018) 1411-1433.

[2] K. Musunuru, F. Sheikh, R.M. Gupta, S.R. Houser, K.O. Maher, D.J. Milan, A. Terzic, J.C. Wu, G. American Heart Association Council on Functional, B. Translational, Y. Council on Cardiovascular Disease in the, C. Council on, N. Stroke, Induced Pluripotent Stem Cells for Cardiovascular Disease Modeling and Precision Medicine: A Scientific Statement From the American Heart Association, Circ Genom Precis Med 11(1) (2018) e000043.

[3] I.Y. Chen, E. Matsa, J.C. Wu, Induced pluripotent stem cells: at the heart of cardiovascular precision medicine, Nat Rev Cardiol 13(6) (2016) 333-49.

[4] Y. Shi, H. Inoue, J.C. Wu, S. Yamanaka, Induced pluripotent stem cell technology: a decade of progress, Nat Rev Drug Discov 16(2) (2017) 115-130.

[5] B.C. Dash, Z. Jiang, C. Suh, Y. Qyang, Induced pluripotent stem cell-derived vascular smooth muscle cells: methods and application, Biochem J 465(2) (2015) 185-94.

[6] G.K. Owens, M.S. Kumar, B.R. Wamhoff, Molecular regulation of vascular smooth muscle cell differentiation in development and disease, Physiol Rev 84(3) (2004) 767-801.

[7] B. Dash, Induced Pluripotent Stem Cell-Derived Vascular Smooth Muscle Cells for Vascular Regeneration, (2020) 20.

[8] C. Patsch, L. Challet-Meylan, E.C. Thoma, E. Urich, T. Heckel, J.F. O'Sullivan, S.J. Grainger, F.G. Kapp, L. Sun, K. Christensen, Y. Xia, M.H. Florido, W. He, W. Pan, M. Prummer, C.R. Warren, R. Jakob-Roetne, U. Certa, R. Jagasia, P.O. Freskgard, I. Adatto, D. Kling, P. Huang, L.I. Zon, E.L. 
Chaikof, R.E. Gerszten, M. Graf, R. Iacone, C.A. Cowan, Generation of vascular endothelial and smooth muscle cells from human pluripotent stem cells, Nat Cell Biol 17(8) (2015) 994-1003.

[9] C. Cheung, A.S. Bernardo, M.W. Trotter, R.A. Pedersen, S. Sinha, Generation of human vascular smooth muscle subtypes provides insight into embryological origin-dependent disease susceptibility, Nat Biotechnol 30(2) (2012) 165-73.

[10] B.C. Dash, K. Levi, J. Schwan, J. Luo, O. Bartulos, H. Wu, C. Qiu, T. Yi, Y. Ren, S. Campbell, M.W. Rolle, Y. Qyang, Tissue-Engineered Vascular Rings from Human iPSC-Derived Smooth Muscle Cells, Stem Cell Reports 7(1) (2016) 19-28.

[11] X. Cong, S.M. Zhang, L. Batty, J. Luo, Application of Human Induced Pluripotent Stem Cells in Generating Tissue-Engineered Blood Vessels as Vascular Grafts, Stem Cells Dev 28(24) (2019) 15811594.

[12] J. Luo, L. Qin, L. Zhao, L. Gui, M.W. Ellis, Y. Huang, M.H. Kural, J.A. Clark, S. Ono, J. Wang, Y. Yuan, S.M. Zhang, X. Cong, G. Li, M. Riaz, C. Lopez, A. Hotta, S. Campbell, G. Tellides, A. Dardik, L.E. Niklason, Y. Qyang, Tissue-Engineered Vascular Grafts with Advanced Mechanical Strength from Human iPSCs, Cell Stem Cell 26(2) (2020) 251-261 e8.

[13] X. Ge, Y. Ren, O. Bartulos, M.Y. Lee, Z. Yue, K.Y. Kim, W. Li, P.J. Amos, E.C. Bozkulak, A. Iyer, W. Zheng, H. Zhao, K.A. Martin, D.N. Kotton, G. Tellides, I.H. Park, L. Yue, Y. Qyang, Modeling supravalvular aortic stenosis syndrome with human induced pluripotent stem cells, Circulation 126(14) (2012) 1695-704.

[14] A. Misra, A.Q. Sheikh, A. Kumar, J. Luo, J. Zhang, R.B. Hinton, L. Smoot, P. Kaplan, Z. Urban, Y. Qyang, G. Tellides, D.M. Greif, Integrin beta3 inhibition is a therapeutic strategy for supravalvular aortic stenosis, J Exp Med 213(3) (2016) 451-63.

[15] J. Bargehr, L. Low, C. Cheung, W.G. Bernard, D. Iyer, M.R. Bennett, L. Gambardella, S. Sinha, Embryological Origin of Human Smooth Muscle Cells Influences Their Ability to Support Endothelial Network Formation, Stem Cells Trans1 Med 5(7) (2016) 946-59. 
[16] B.C. Dash, O. Setia, J. Gorecka, H. Peyvandi, K. Duan, L. Lopes, J. Nie, F. Berthiaume, A. Dardik,

H.C. Hsia, A Dense Fibrillar Collagen Scaffold Differentially Modulates Secretory Function of iPSCDerived Vascular Smooth Muscle Cells to Promote Wound Healing, Cells 9(4) (2020).

[17] J. Gorecka, X. Gao, A. Fereydooni, B.C. Dash, J. Luo, S.R. Lee, R. Taniguchi, H.C. Hsia, Y. Qyang, A. Dardik, Induced pluripotent stem cell-derived smooth muscle cells increase angiogenesis and accelerate diabetic wound healing, Regen Med 15(2) (2020) 1277-1293.

[18] L. Daneshmandi, S. Shah, T. Jafari, M. Bhattacharjee, D. Momah, N. Saveh-Shemshaki, K.W. Lo, C.T. Laurencin, Emergence of the Stem Cell Secretome in Regenerative Engineering, Trends Biotechnol 38(12) (2020) 1373-1384.

[19] P. Dhavalikar, A. Robinson, Z. Lan, D. Jenkins, M. Chwatko, K. Salhadar, A. Jose, R. Kar, E. Shoga, A. Kannapiran, E. Cosgriff-Hernandez, Review of Integrin-Targeting Biomaterials in Tissue Engineering, Adv Healthc Mater (2020) e2000795.

[20] M.R. Sprott, G. Gallego-Ferrer, M.J. Dalby, M. Salmeron-Sanchez, M. Cantini, Functionalization of PLLA with Polymer Brushes to Trigger the Assembly of Fibronectin into Nanonetworks, Adv Healthc Mater 8(3) (2019) e1801469.

[21] S. Trujillo, S.L. Vega, K.H. Song, A. San Felix, M.J. Dalby, J.A. Burdick, M. Salmeron-Sanchez, Engineered Full-Length Fibronectin-Hyaluronic Acid Hydrogels for Stem Cell Engineering, Adv Healthc Mater 9(21) (2020) e2000989.

[22] S. Trujillo, C. Gonzalez-Garcia, P. Rico, A. Reid, J. Windmill, M.J. Dalby, M. Salmeron-Sanchez, Engineered 3D hydrogels with full-length fibronectin that sequester and present growth factors, Biomaterials 252 (2020) 120104.

[23] D.L. Brassard, E. Maxwell, M. Malkowski, T.L. Nagabhushan, C.C. Kumar, L. Armstrong, Integrin alpha(v)beta(3)-mediated activation of apoptosis, Exp Cell Res 251(1) (1999) 33-45. 
[24] W. Wang, F. Wang, F. Lu, S. Xu, W. Hu, J. Huang, Q. Gu, X. Sun, The antiangiogenic effects of integrin alpha5beta1 inhibitor (ATN-161) in vitro and in vivo, Invest Ophthalmol Vis Sci 52(10) (2011) 7213-20.

[25] B.C. Dash, K. Duan, H. Xing, T.R. Kyriakides, H.C. Hsia, An in situ collagen-HA hydrogel system promotes survival and preserves the proangiogenic secretion of hiPSC-derived vascular smooth muscle cells, Biotechnol Bioeng (2020).

[26] K.L. DeCicco-Skinner, G.H. Henry, C. Cataisson, T. Tabib, J.C. Gwilliam, N.J. Watson, E.M. Bullwinkle, L. Falkenburg, R.C. O'Neill, A. Morin, J.S. Wiest, Endothelial cell tube formation assay for the in vitro study of angiogenesis, J Vis Exp (91) (2014) e51312.

[27] A. Granata, F. Serrano, W.G. Bernard, M. McNamara, L. Low, P. Sastry, S. Sinha, An iPSCderived vascular model of Marfan syndrome identifies key mediators of smooth muscle cell death, Nat Genet 49(1) (2017) 97-109.

[28] L. Gui, B.C. Dash, J. Luo, L. Qin, L. Zhao, K. Yamamoto, T. Hashimoto, H. Wu, A. Dardik, G. Tellides, L.E. Niklason, Y. Qyang, Implantable tissue-engineered blood vessels from human induced pluripotent stem cells, Biomaterials 102 (2016) 120-9.

[29] A.Y. Clark, K.E. Martin, J.R. Garcia, C.T. Johnson, H.S. Theriault, W.M. Han, D.W. Zhou, E.A. Botchwey, A.J. Garcia, Integrin-specific hydrogels modulate transplanted human bone marrow-derived mesenchymal stem cell survival, engraftment, and reparative activities, Nat Commun 11(1) (2020) 114.

[30] B.G. Keselowsky, D.M. Collard, A.J. Garcia, Integrin binding specificity regulates biomaterial surface chemistry effects on cell differentiation, Proc Natl Acad Sci U S A 102(17) (2005) 5953-7.

[31] M. Presta, P. Dell'Era, S. Mitola, E. Moroni, R. Ronca, M. Rusnati, Fibroblast growth factor/fibroblast growth factor receptor system in angiogenesis, Cytokine Growth Factor Rev 16(2) (2005) 159-78.

[32] M. Murakami, M. Simons, Fibroblast growth factor regulation of neovascularization, Curr Opin Hematol 15(3) (2008) 215-20. 
[33] M. Rusnati, E. Tanghetti, P. Dell'Era, A. Gualandris, M. Presta, alphavbeta3 integrin mediates the cell-adhesive capacity and biological activity of basic fibroblast growth factor (FGF-2) in cultured endothelial cells, Mol Biol Cell 8(12) (1997) 2449-61.

[34] M.D. Sternlicht, Z. Werb, How matrix metalloproteinases regulate cell behavior, Annu Rev Cell Dev Biol 17 (2001) 463-516.

[35] H. Nagase, R. Visse, G. Murphy, Structure and function of matrix metalloproteinases and TIMPs, Cardiovasc Res 69(3) (2006) 562-73.

[36] M. Rolli, E. Fransvea, J. Pilch, A. Saven, B. Felding-Habermann, Activated integrin alphavbeta3 cooperates with metalloproteinase MMP-9 in regulating migration of metastatic breast cancer cells, Proc Natl Acad Sci U S A 100(16) (2003) 9482-7.

[37] P.A. Rupp, R.P. Visconti, A. Czirok, D.A. Cheresh, C.D. Little, Matrix metalloproteinase 2-integrin alpha(v)beta3 binding is required for mesenchymal cell invasive activity but not epithelial locomotion: a computational time-lapse study, Mol Biol Cell 19(12) (2008) 5529-40.

[38] P.C. Brooks, S. Stromblad, L.C. Sanders, T.L. von Schalscha, R.T. Aimes, W.G. Stetler-Stevenson, J.P. Quigley, D.A. Cheresh, Localization of matrix metalloproteinase MMP-2 to the surface of invasive cells by interaction with integrin alpha v beta 3, Cell 85(5) (1996) 683-93.

[39] U.B. Hofmann, J.R. Westphal, A.A. Van Kraats, D.J. Ruiter, G.N. Van Muijen, Expression of integrin alpha(v)beta(3) correlates with activation of membrane-type matrix metalloproteinase-1 (MT1MMP) and matrix metalloproteinase-2 (MMP-2) in human melanoma cells in vitro and in vivo, Int J Cancer 87(1) (2000) 12-9.

[40] Y. Jiao, X. Feng, Y. Zhan, R. Wang, S. Zheng, W. Liu, X. Zeng, Matrix metalloproteinase-2 promotes alphavbeta3 integrin-mediated adhesion and migration of human melanoma cells by cleaving fibronectin, PLoS One 7(7) (2012) e41591. 
[41] E. Levi, R. Fridman, H.Q. Miao, Y.S. Ma, A. Yayon, I. Vlodavsky, Matrix metalloproteinase 2 releases active soluble ectodomain of fibroblast growth factor receptor 1, Proc Natl Acad Sci U S A 93(14) (1996) 7069-74.

[42] V.A. Belo, D.A. Guimaraes, M.M. Castro, Matrix Metalloproteinase 2 as a Potential Mediator of Vascular Smooth Muscle Cell Migration and Chronic Vascular Remodeling in Hypertension, J Vasc Res 52(4) (2015) 221-31.

[43] A.A. Troussard, C. Tan, T.N. Yoganathan, S. Dedhar, Cell-extracellular matrix interactions stimulate the AP-1 transcription factor in an integrin-linked kinase- and glycogen synthase kinase 3dependent manner, Mol Cell Biol 19(11) (1999) 7420-7.

[44] E. Toschi, G. Barillari, C. Sgadari, I. Bacigalupo, A. Cereseto, D. Carlei, C. Palladino, C. Zietz, P. Leone, M. Sturzl, S. Butto, A. Cafaro, P. Monini, B. Ensoli, Activation of matrix-metalloproteinase-2 and membrane-type-1-matrix-metalloproteinase in endothelial cells and induction of vascular permeability in vivo by human immunodeficiency virus-1 Tat protein and basic fibroblast growth factor, Mol Biol Cell 12(10) (2001) 2934-46.

[45] K. Forbes, M.A. Webb, I. Sehgal, Growth factor regulation of secreted matrix metalloproteinase and plasminogen activators in prostate cancer cells, normal prostate fibroblasts and normal osteoblasts, Prostate Cancer Prostatic Dis 6(2) (2003) 148-53. 


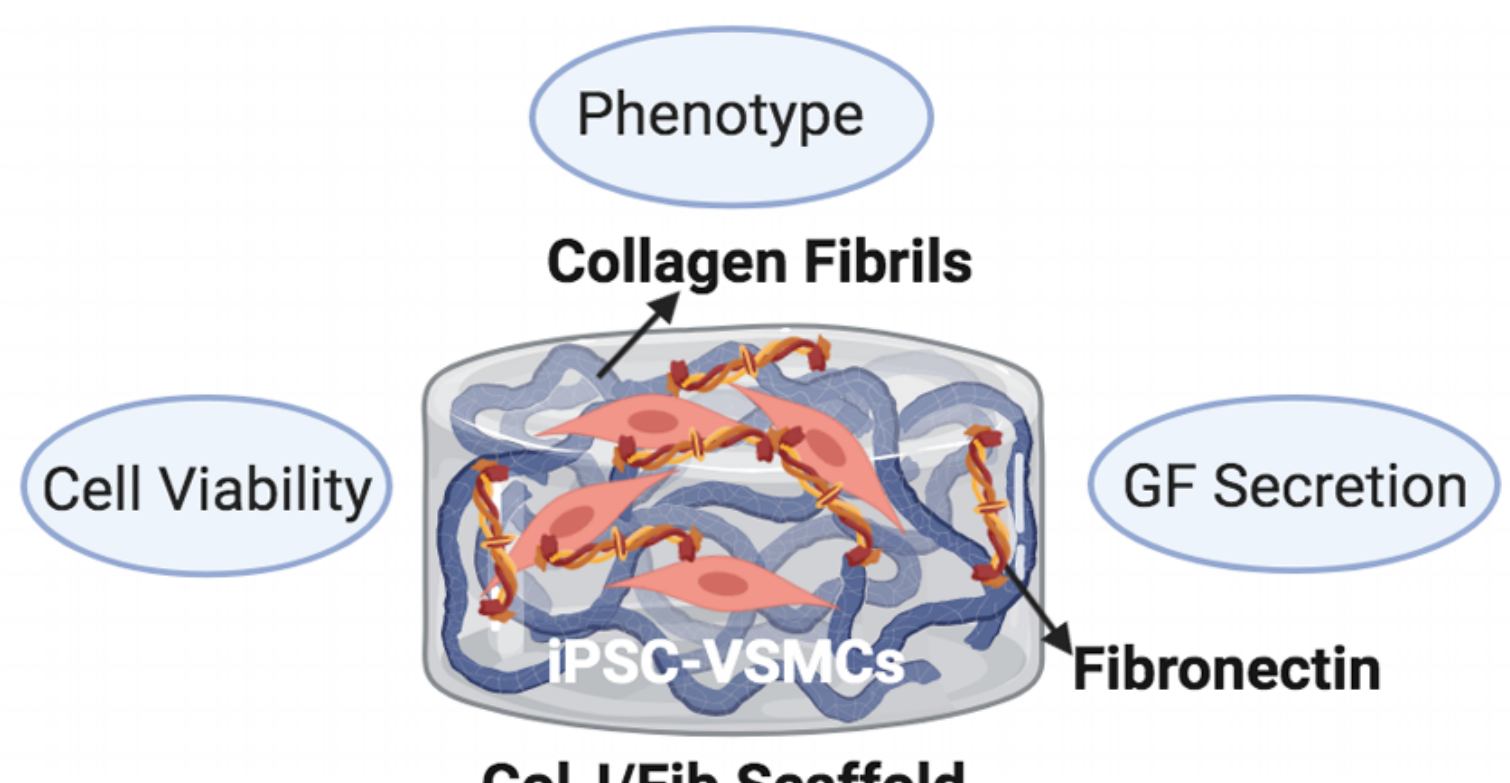

Col-I/Fib Scaffold

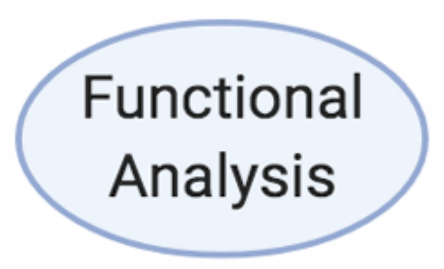

Figure 1: Schematic showing the fabrication and characterization of a Collagen/Fibronectin Scaffold for the modulation of hiPSC-VSMCs proangiogenic factor. Created with BioRender.com 

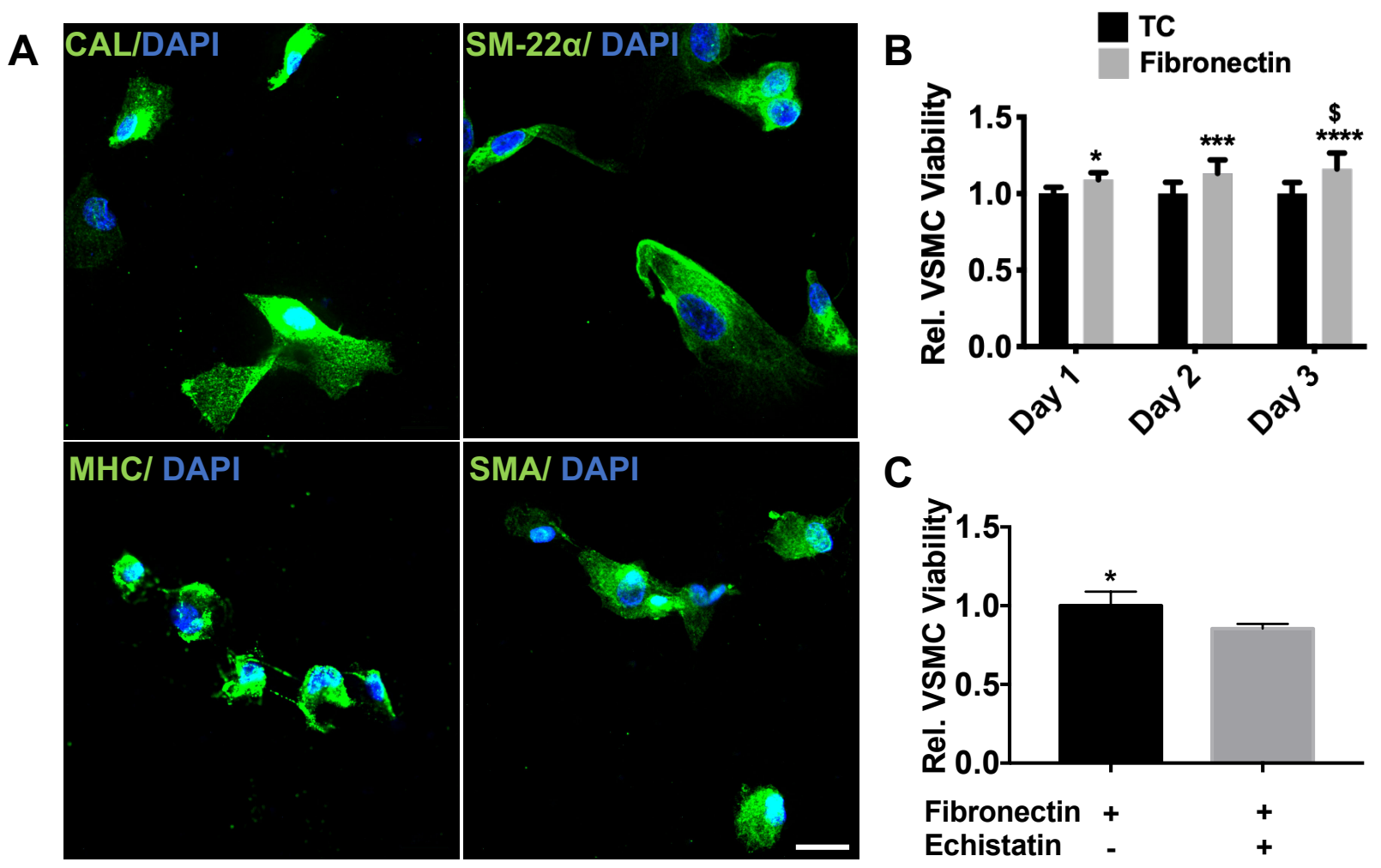

Figure 2: 2D characterization of the effect of fibronectin on hiPSC-VSMC's viability. (A) The hiPSCVSMCs differentiated using an embryoid body method were characterized using smooth muscle cell markers. Phenotype assessment of hiPSC-VSMCs were performed using immunofluorescence staining with Calponin, SM-22 $\alpha$, smooth muscle myosin heavy chain (SM-MHC) and smooth muscle $\alpha$ actin (SMA) in Green and Dapi stained nuclei in Blue. Scale bar measures $20 \mu \mathrm{m}$. AlamarBlue assay showing relative cell viability of hiPSC-VSMCs (B) with and without fibronectin coating for day 1, 2 and 3 and (C) with and without $\alpha_{\mathrm{V}} \beta_{3}$ antagonist echistatin $(100 \mathrm{nM} / \mathrm{mL})$ on fibronectin coated plates on day $3 . *$ denotes statistical significance differences between the different groups ( $\mathrm{n}=4-12$, one-way and two-way ANOVA, $* \mathrm{p}<0.05, * * * \mathrm{p}<0.001$, and $* * * * \mathrm{p}<0.0001)$. 

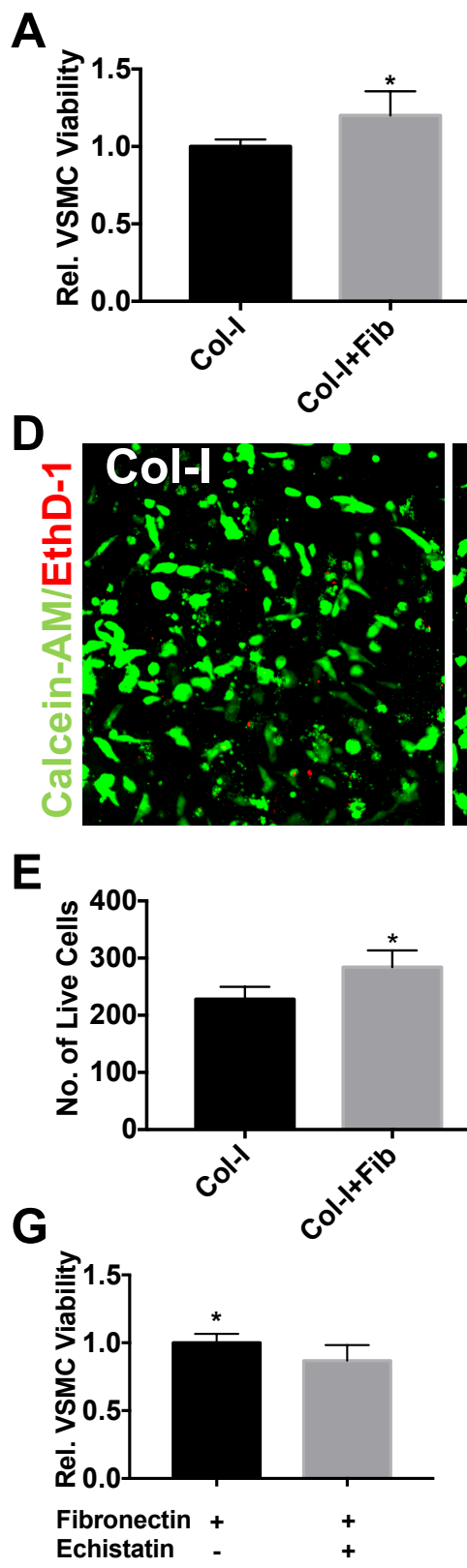

B
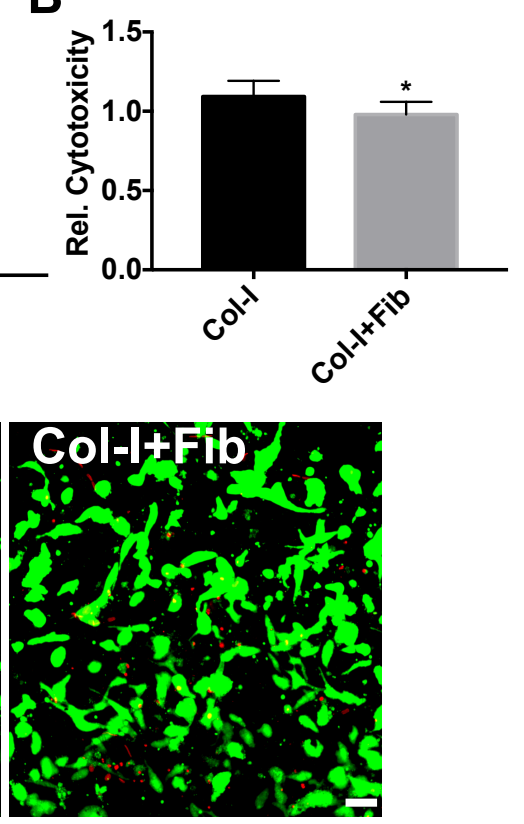

F
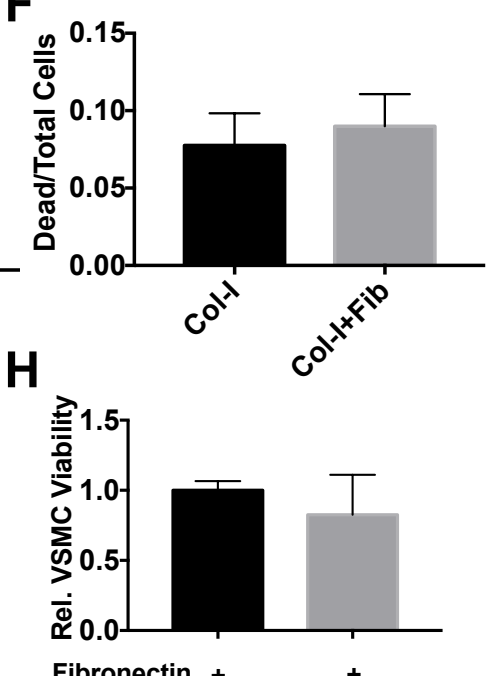
ATN-161

C
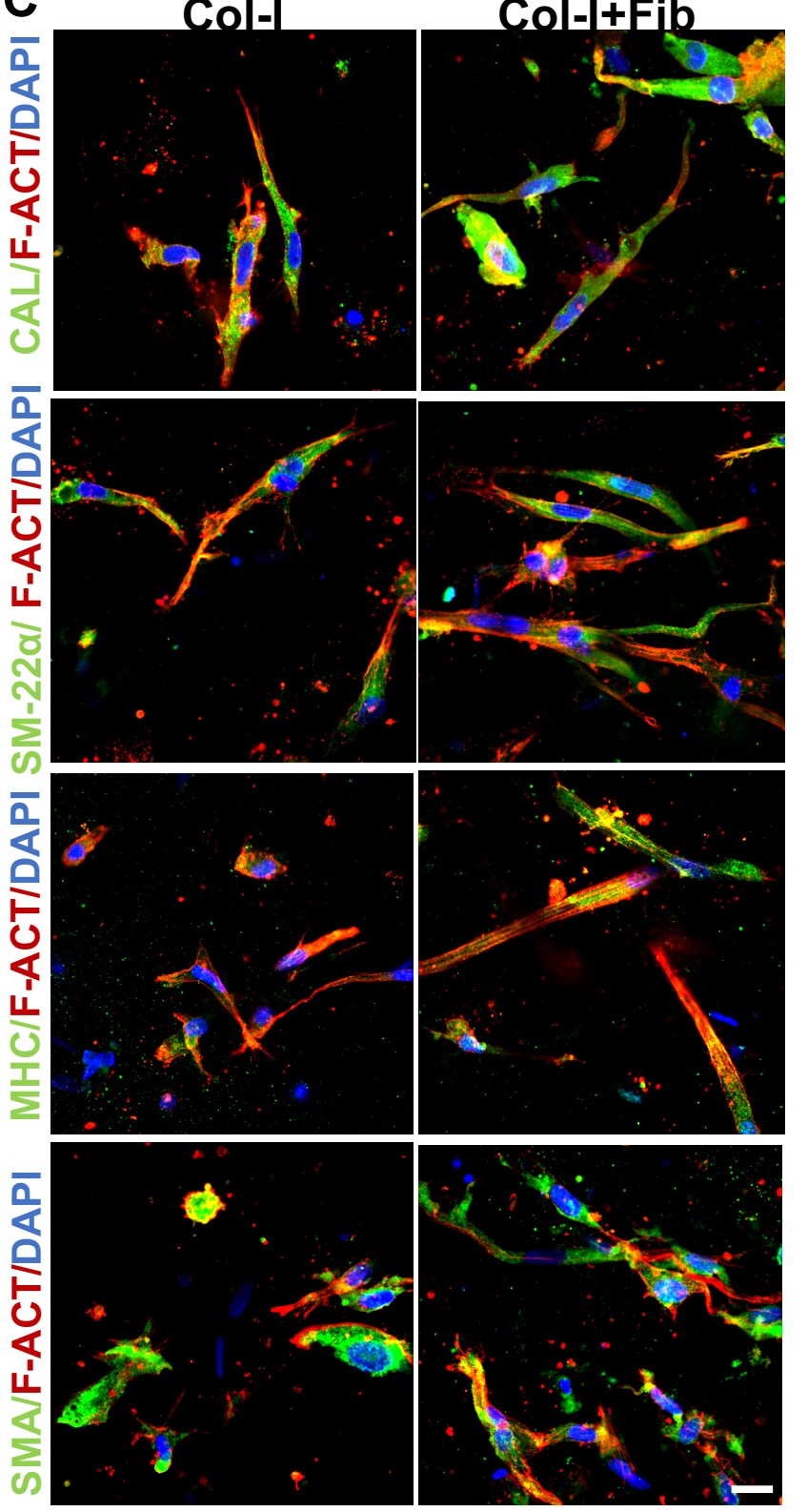

Figure 3: Characterization of hiPSC-VSMC viability and phenotype embedded in the collagen/fibronectin scaffold. (A) AlamarBlue cell viability assay showing relative cell viability of hiPSC-VSMCs embedded in the collagen/fibronectin scaffolds of $4 \mathrm{mg} / \mathrm{ml}$ of collagen concentration. (B) LDH assay of conditioned medium of the collagen/fibronectin scaffolds. (C) Phenotype assessment of hiPSC-VSMCs embedded in the in situ hydrogels. Immunofluorescence images showing calponin, SM- 
$22 \alpha$, smooth muscle myosin heavy chain (SM-MHC) and smooth muscle $\alpha$ actin (SMA) in Green and Dapi stained nuclei in Blue. Scale bar measures $20 \mu \mathrm{m}$. (D) Live/Dead assay of the collagen/fibronectin scaffold. Calcein-AM and EthD-1 stains live cells (Green) and dead cells (Red) respectively. Scale bar measures 50 $\mu \mathrm{m}$. The graph shows (E) the number of Calcein-AM positive live (green) cells and (F) the ratio of dead/total cells. Collagen scaffolds without fibronectin were kept as controls for all the experiments. AlamarBlue assay showing relative cell viability of hiPSC-VSMCs in collagen/fibronectin scaffolds with and without $(\mathrm{G}) \alpha_{\mathrm{V}} \beta_{3}$ antagonist echistatin and $(\mathrm{H}) \alpha_{5} \beta_{1}$ antagonist ATN-161. * denotes statistical significance differences between the different groups $\left(n=3-6\right.$, t-test, $\left.{ }^{*} \mathrm{p}<0.05\right)$. 

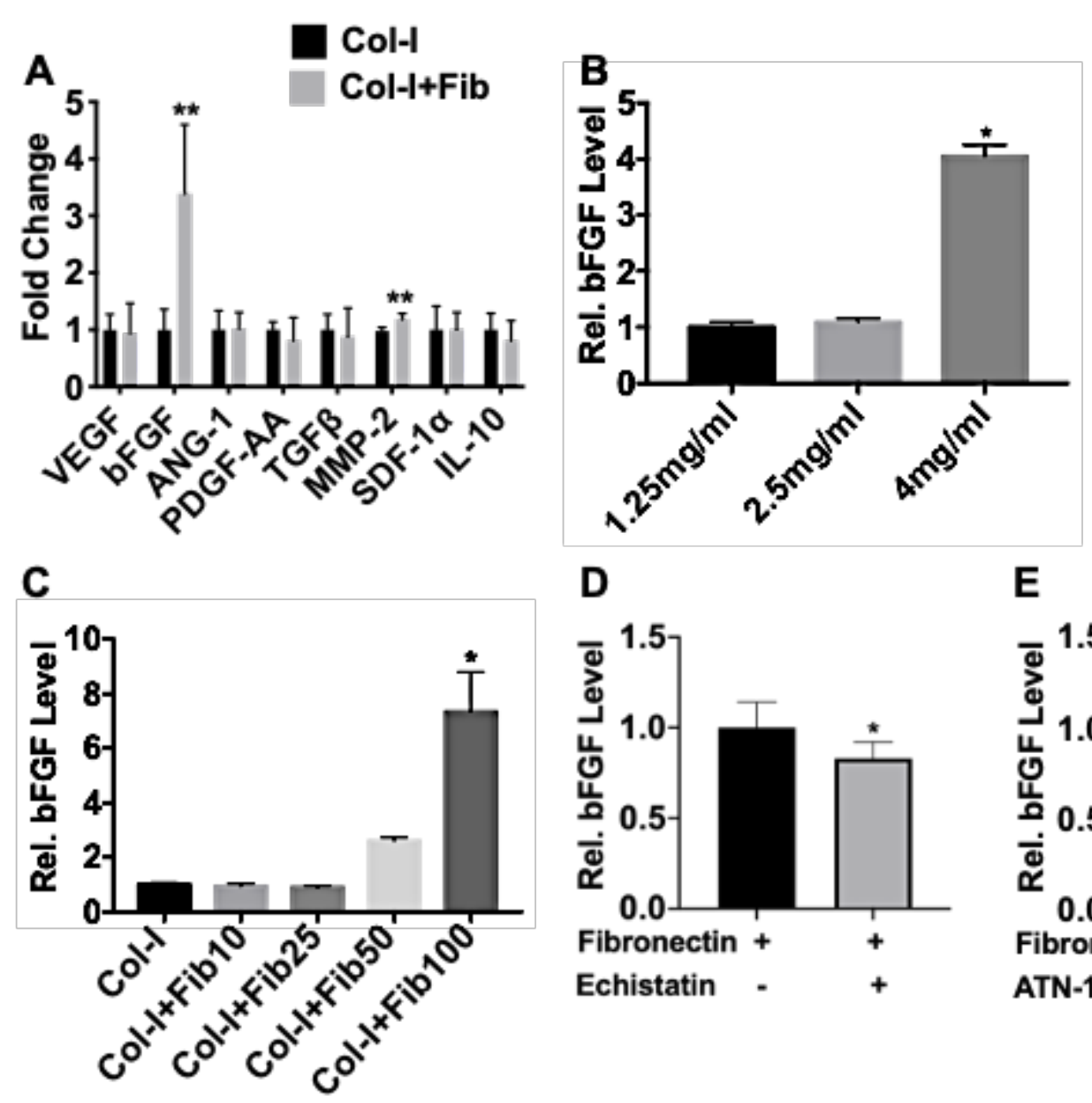

E

Figure 4: Characterization of pro-angiogenic growth factor secretion from the collagen/fibronectin scaffolds. (A) Qualitative ELISA showing secretion of relative levels of growth factors VEGF, bFGF, ANG-1, PDGF-AA, TGF $\beta$, MMP-2, SDF-1 $\alpha$, and IL-10. ELISA data showing relative bFGF level in (B) response to fibronectin $(50 \mu \mathrm{g} / \mathrm{ml})$ in various densities of collagen scaffold, and (C) different dosages (5, $10,25,50$ and $100 \mu \mathrm{g} / \mathrm{ml}$ ) of fibronectin in a $4 \mathrm{mg} / \mathrm{ml}$ density of collagen scaffold. Inhibition assay with with and without (D) echistatin and (E) ATN-161 in a 4mg/ml density of collagen scaffold with $100 \mu \mathrm{g} / \mathrm{ml}$ of fibronectin. Collagen scaffolds with and without fibronectin were kept as controls. * denotes statistical significance differences between the different groups $\left(\mathrm{n}=3-6, \mathrm{t}\right.$-test and one-way ANOVA, ${ }^{*} \mathrm{p}<0.05$, $* * \mathrm{p}<0.01)$. 

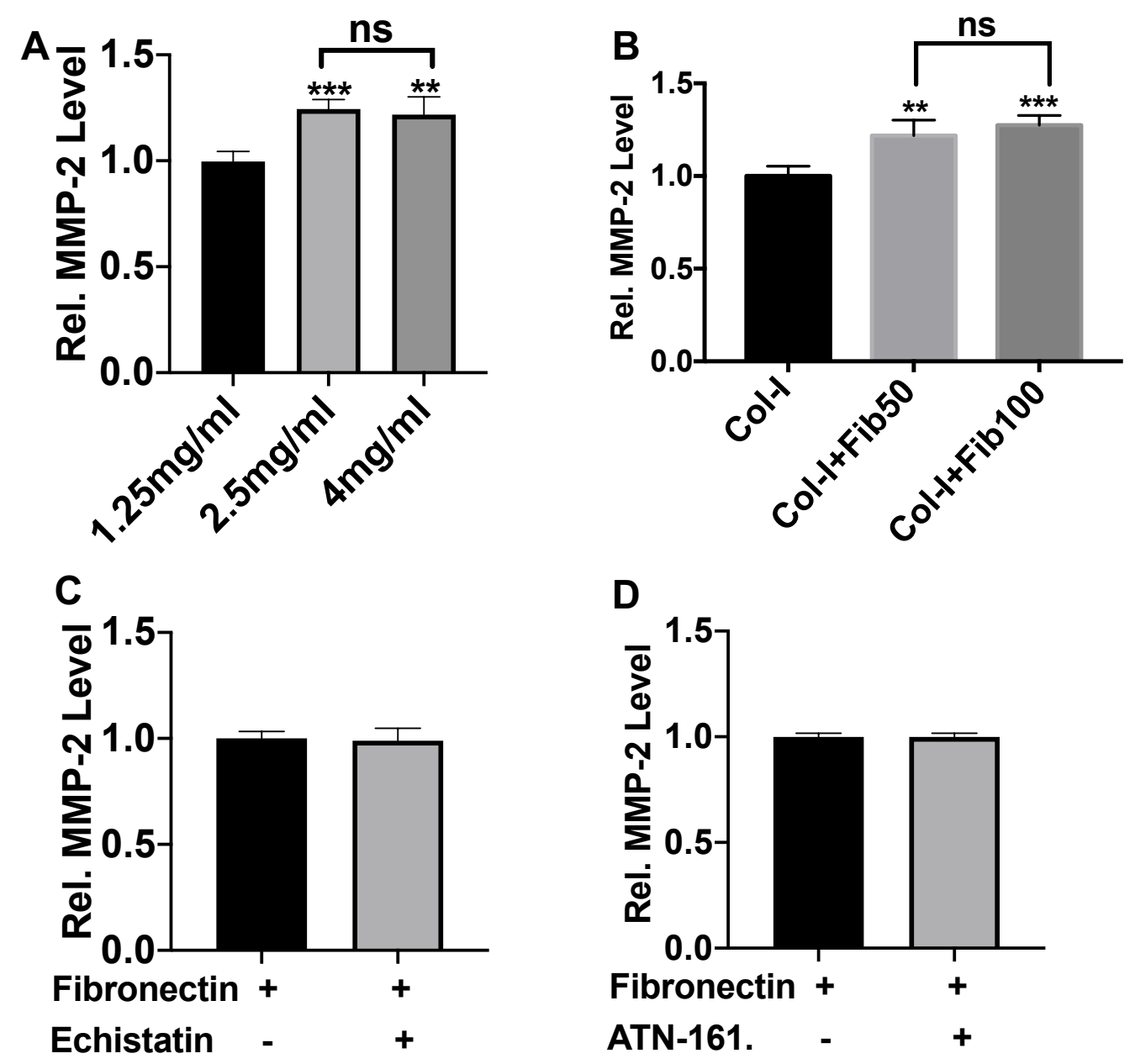

Figure 5: Characterization of MMP-2 secretion. ELISA data showing relative MMP-2 level in response to (A) fibronectin $(50 \mu \mathrm{g} / \mathrm{ml})$ in various densities of $4 \mathrm{mg} / \mathrm{ml}$ collagen scaffold, (B) different dosages (50 and $100 \mu \mathrm{g} / \mathrm{ml}$ ) of fibronectin in a $4 \mathrm{mg} / \mathrm{ml}$ collagen scaffold, (C) incubation with Echistatin and (D) ATN161. Collagen scaffolds with and without fibronectin were kept as controls. * denotes statistical significance differences between the different groups $(\mathrm{n}=3-6, \mathrm{t}$-test and one-way ANOVA, $* * \mathrm{p}<0.01$, $* * * \mathrm{p}<0.001)$. 

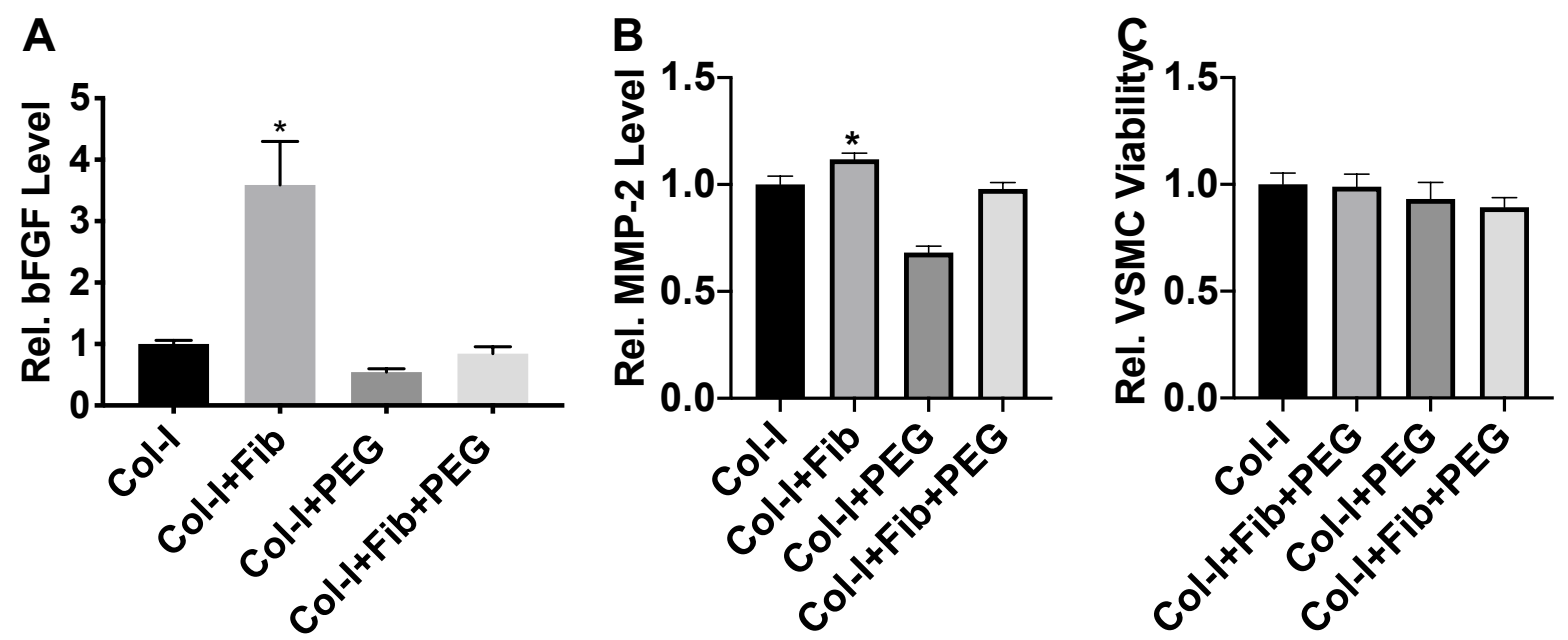

Figure 6: Characterization of the effect of cross-linking on bFGF and MMP-2 secretion. Qualitative ELISA showing secretion of relative level of (A) bFGF and (B) MMP-2 in various formulation of collagen hydrogel: collagen only (Col-I), fibronectin functionalized collagen gel (Col-I+Fib), 4S-StarPEG crosslinked collagen gel (Col-I+PEG) and 4S-StarPEG cross-linked collagen and fibronectin gel (ColI+Fib+PEG). AlamarBlue assay showing viability of hiPSC-VSMC in aforementioned collagen hydrogel formulations. * denotes statistical significance differences between the different groups $(\mathrm{n}=4$, one-way ANOVA, $\left.{ }^{*} \mathrm{p}<0.05\right)$. 

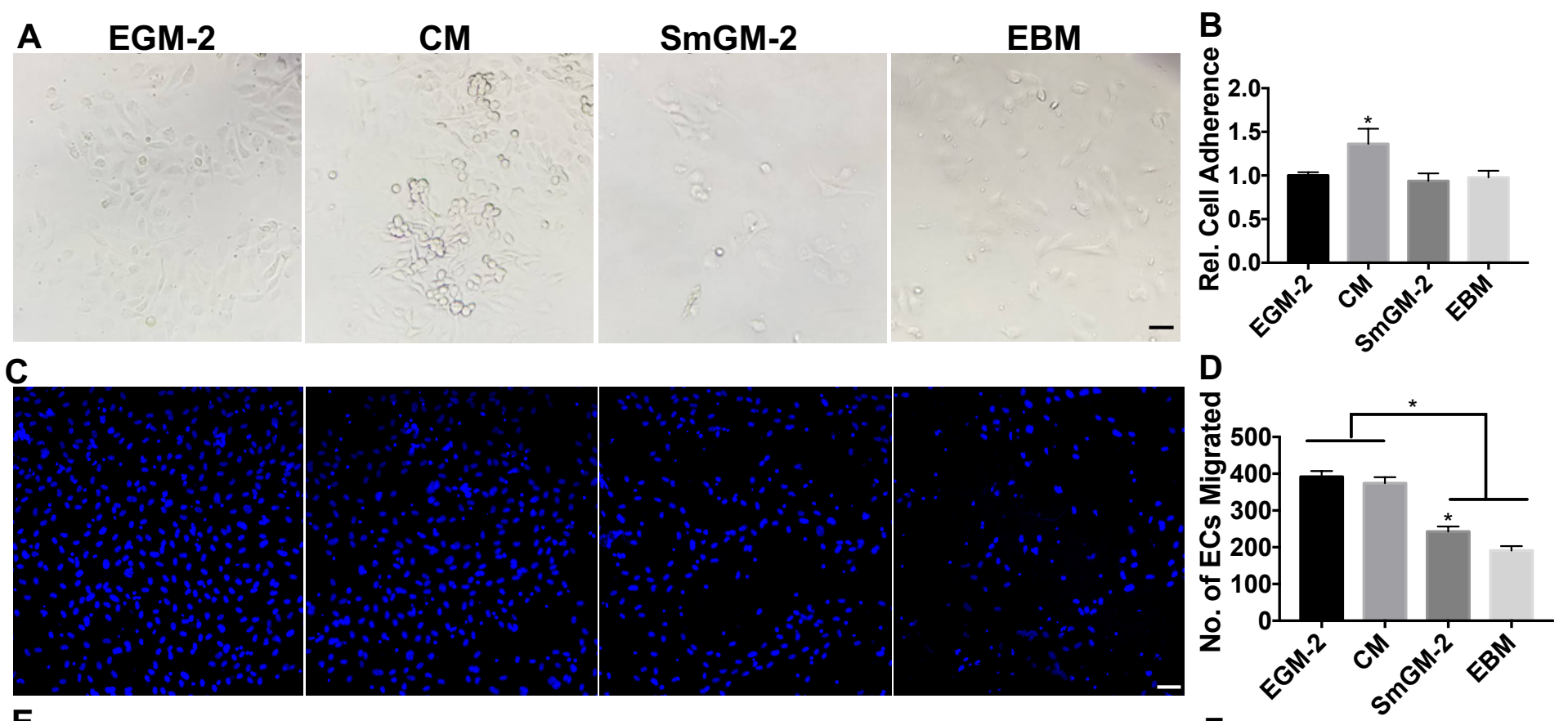

E
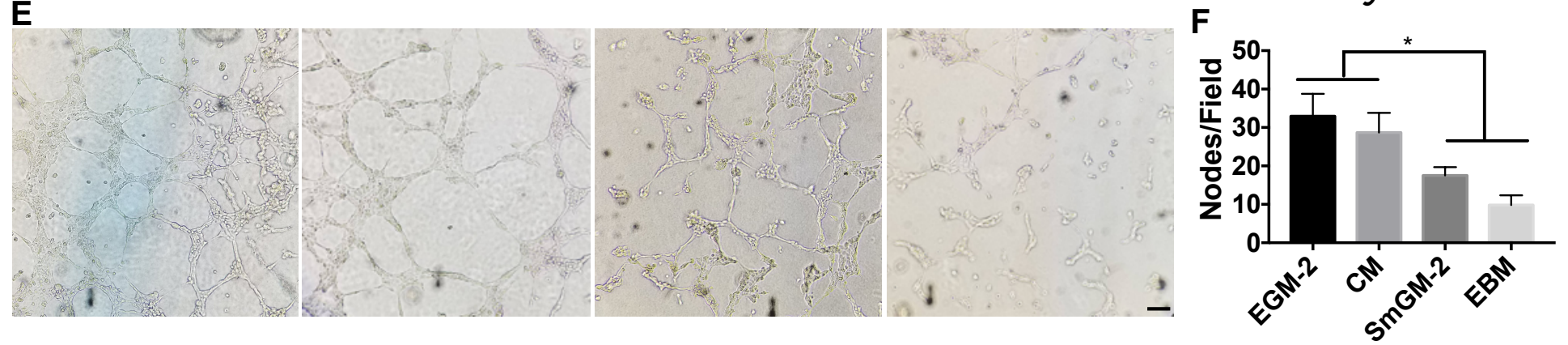

Figure 7: Functional assessment of the conditioned medium from collagen/fibronectin scaffolds. The

conditioned medium (CM) collected from the collagen/fibronectin scaffolds were tested for their proangiogenic bioactivity using HUVEC. (A) Brightfield images showing attachment of HUVEC on 0.1\% coated gelatin wells after $3 \mathrm{hr}$ of seeding. Scale bar measures $100 \mu \mathrm{m}$. (B) AlamarBlue assay was performed to determine relative level of HUVEC adherence on gelatin coated plate after $3 \mathrm{hr}$ of seeding. Eight micrometer pore size trans-wells were used to determine migration of HUVECs in response to the CM over $4 \mathrm{hr}$ of incubation time. (C) The cells migrated were stained with dapi and (D) counted to obtain the total number of cells migrated. Scale bar measures $50 \mu \mathrm{m}$. Angiogenesis assay was performed using Matrigel and HUVEC. (E) Brightfield images showing network formation of HUVEC in response to CM from in situ collagen/fibronectin scaffolds after $6 \mathrm{~h}$ of incubation. Scale bar measures $100 \mu \mathrm{m}$. (F) The graph represents number of nodes/field. Endothelial cell (EGM-2) and smooth muscle cell (SmGM-2) growth 
bioRxiv preprint doi: https://doi.org/10.1101/2021.01.06.425573; this version posted January 6, 2021. The copyright holder for this preprint (which was not certified by peer review) is the author/funder. All rights reserved. No reuse allowed without permission.

medium, and endothelial cell basal medium (EBM) were used as controls. * denotes statistical significance differences between the different groups ( $\mathrm{n}=3-6$, one-way ANOVA, $\left.{ }^{*} \mathrm{p}<0.05\right)$. 


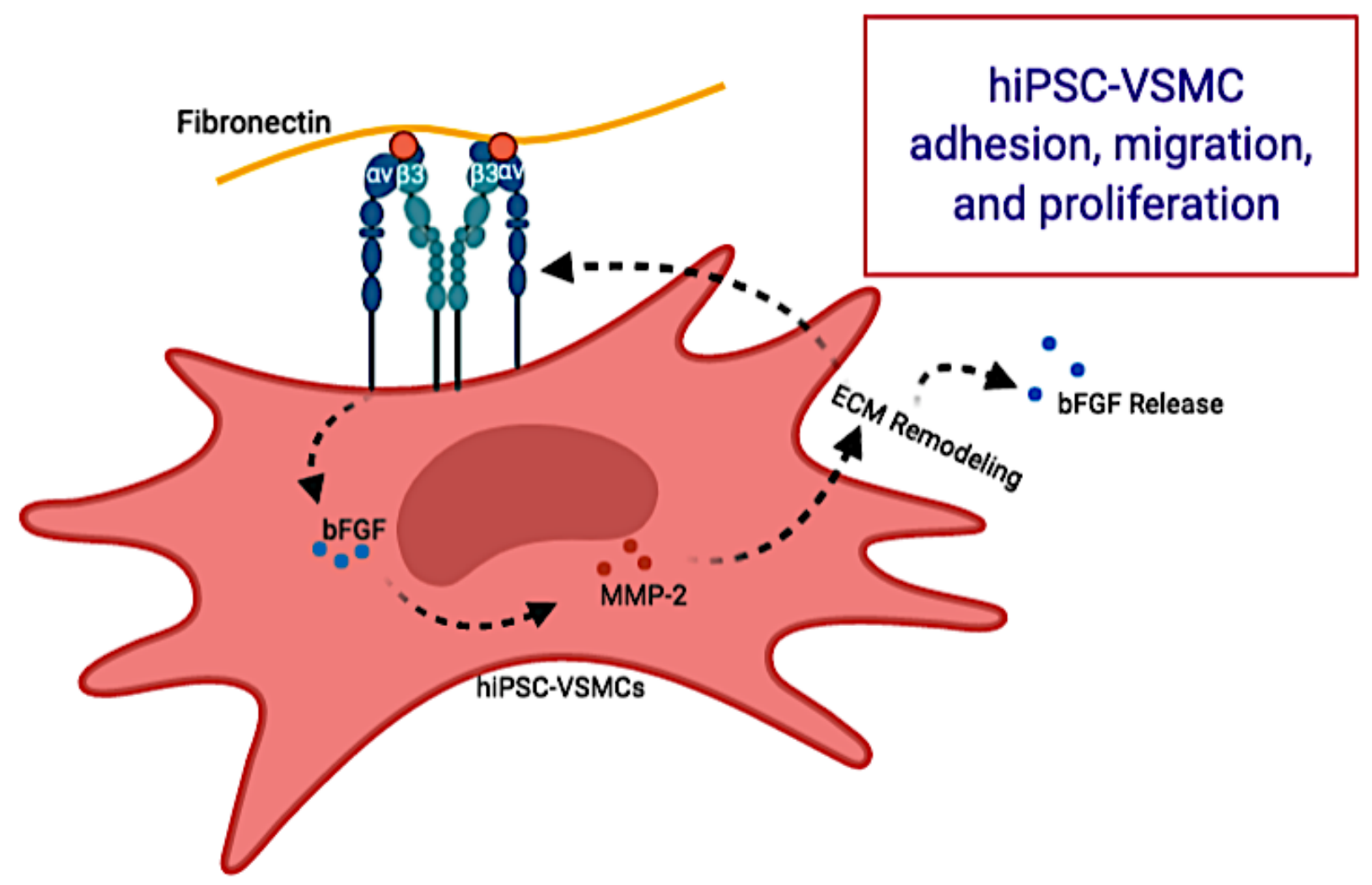

Figure 8: Schematic showing integrin $\beta_{3}$, bFGF and MMP-2 positive feedback loop system modulating hiPSC-VSMC function. Created with BioRender.com 